\title{
Intermediate Ischemic Risk
}

National Cancer Institute

\section{Source}

National Cancer Institute. Intermediate Ischemic Risk. NCI Thesaurus. Code C99967.

The likelihood an individual will develop ischemia is moderate. (ACC) 